\title{
Design Criteria of a Helmholtz Silencer with Multiple Degrees of Freedom for Hydraulic Systems*
}

\author{
Takayoshi ICHIYANAGI**, Tetsuya KURIBAYASHI*** \\ and Takao NISHIUMI** \\ ** Department of Mechanical Systems Engineering, National Defense Academy, \\ 1-10-20 Hashirimizu, Yokosuka, Kanagawa, 239-8686, Japan \\ E-mail: ichiyana@nda.ac.jp \\ *** Graduate School of Science and Engineering, National Defense Academy
}

\begin{abstract}
The vibration and noise caused by pressure pulsation, referred to as fluid-borne vibration or fluid-borne noise, are some of the most detrimental problems in hydraulic systems. A Helmholtz silencer with multiple degrees of freedom was proposed to attenuate several harmonic frequencies generated in the hydraulic systems. The silencer consists of a cylindrical vessel with several chokes inside the vessel. The final goal of this research project is the development of a multiple-degree-of-freedom type of Helmholtz silencer that can be applied to hydraulic pump systems operated at different speeds. The aim of this report is to establish the design criteria of the silencer specifications. In particular, the effects of the diameter and length of the choke and the cylindrical volume on attenuation performance were analytically and experimentally investigated.
\end{abstract}

Key words : Pressure Pulsation, Helmholtz Silencer, Hydraulic System, Transmission Loss, Fluid-Borne Noise

\section{Introduction}

A positive displacement pump generates flow pulsation that interacts with a hydraulic circuit to produce pressure pulsation. Pressure pulsation is usually the primary noise source in hydraulic circuits because it is very easily transmitted throughout the entire system and excites mechanical parts that generate audible noise. The pressure pulsation is a periodic function of time and its frequency characteristics are composed of a fundamental frequency and higher harmonics. The amplitudes of the first, second, and third harmonics are often dominant components in pressure pulsation. Therefore, attenuating the amplitude of these harmonics is an effective way to reduce the system noise. The Helmholtz type silencer is a classic device for attenuating oscillations such as audible noise; it is also utilized in hydraulic systems to attenuate the pressure pulsation ${ }^{(1)}$. A distinctive attenuation characteristic of the Helmholtz silencer is that the effective range of attenuation frequency is narrow. Therefore, the pressure pulsation cannot be sufficiently attenuated if the pumping frequency is not within the effective attenuation range of the silencer. A hydraulic silencer, referred to as the multiple-degree-of-freedom Helmholtz silencer (MHS), has been previously developed to attenuate the amplitude of several harmonics of pressure pulsation (2). Unlike the conventional Helmholtz hydraulic silencer, this device incorporates a feature that allows the resonance mode to be adjusted freely as desired and is a single closed-end cylindrical vessel. Because of this silencer's compactness and attenuation ability, it is 
suitable for use in hydraulic systems running at constant pump rotational speeds such as excavators and injection molding machines. However, the problems associated with narrow band attenuation characteristics remain if the hydraulic system is operated with a variable rotation speed pump. In this case, a silencer that covers a wider frequency range is desired. Consequently, the final goal of this research project is to apply the MHS to a hydraulic system operated under variable pump speed conditions. This will be achieved by adding a variable resonance mechanism to the silencer. In order to design a silencer that attains the desired resonance mode in practice, the design criteria are indispensable in the determination of appropriate silencer dimensions. In a previous research, the MHS was modeled using a distributed parameter model and all dimensions were determined using the numerical optimum design method ${ }^{(2)}$. Hence, the design parameters could not be estimated in advance. For a MHS with variable resonant ability, the computation cost of the numerical optimum design method will become enormous unless some dimensions are constrained to decrease the design variables of the numerical calculation. Therefore, this paper discusses correlations between the silencer dimensions and attenuation characteristics in order to obtain the design criteria for the MHS with variable resonant ability. The lumped parameter models for both multiple-degree-of-freedom and conventional types are described and used for the design parametric study. In the past, many studies have attempted to clarify the attenuation characteristics of the conventional Helmholtz silencer for both the lumped and distributed parameter models ${ }^{(3)-(8)}$. In these researches, the influence of geometry on the resonant frequency has been already well discussed. However, most of them were for the acoustic noise attenuator that can disregard the viscosity effect of fluid. The property of hydraulic oil was also different from that of air. Besides, the parametric study for the peak value of transmission loss was not examined yet. This paper focuses on acquiring knowledge about the relationship between the silencer dimensions and attenuation performance with respect to the resonant frequency and transmission loss. This knowledge is applied to the initial design of the MHS with variable resonant ability. In addition to the theoretical parametric study, experimental work is conducted to verify the calculated results. Finally, a prototype design of a three-degree-of-freedom Helmholtz silencer (3HS) is described to evaluate the feasibility of variable resonant ability.

\section{Nomenclature}

$\begin{array}{ll}a_{i} & : \text { Cross-sectional area of the } i \text { th choke } \\ c & : \text { Speed of sound } \\ C_{i} & : \text { Damping coefficient of the } i \text { th choke } \\ D_{0} & : \text { Diameter of volume of the present Helmholtz silencer } \\ D_{h} & : \text { Diameter of volume of the conventional Helmholtz silencer } \\ d_{h} & : \text { Diameter of choke of the conventional Helmholtz silencer } \\ d_{i} & : \text { Diameter of the } i \text { th choke } \\ d_{0} & : \text { Diameter of the main line } \\ f_{n} & : \text { Resonant frequency } \\ f_{r . i} & : \text { ith resonant frequency of silencer } \\ f_{r . i}^{*} & : \text { Desired value of } f_{r . i} \\ H & : \text { Coefficient of entrance impedance } \\ K & : \text { Gain constant } \\ k_{i} & : \text { Spring coefficient of the } i \text { th volume }\end{array}$


$L_{i} \quad$ : Length of the $i$ th volume

$L_{h} \quad$ : Length of volume of the conventional Helmholtz silencer

$l_{c} \quad:$ Length of choke of the conventional Helmholtz silencer used in the experiment

$l_{i} \quad$ : Length of the $i$ th choke

$l_{h} \quad:$ Length of choke of the conventional Helmholtz silencer

$l_{p 1 \sim p 4} \quad:$ Length between the pressure transducers and silencer

$m_{i} \quad$ : Mass of the fluid in the $i$ th choke

$M \quad$ : Coefficient of entrance impedance

$P \quad$ : Pressure pulsation

$Q \quad$ : Flow pulsation

$s \quad$ : Laplace operator

TL : Transmission loss

$V_{h} \quad:$ Volume of the conventional Helmholtz silencer

$V_{i} \quad: i$ th volume

$W_{i} \quad$ : Incident energy of pulsation into silencer

$W_{t} \quad$ : Transmitted energy of pulsation through silencer

$\mathbf{X} \quad$ : Design variables

$Z_{c} \quad:$ Characteristic impedance of the main line

$Z_{h} \quad:$ Entrance impedance of the silencer

$\beta \quad$ : Open-end correction coefficient

$\varepsilon \quad: \quad$ Ratio of transmission loss peak

$\kappa \quad$ : Bulk modulus of oil

$v \quad$ : Kinematic viscosity of oil

$\rho \quad$ : Density of oil

$\omega_{n} \quad$ : Resonant angular frequency of the conventional Helmholtz silencer

$\xi \quad$ : Complex coefficient for unsteady viscous friction effect

$\zeta \quad$ : Damping coefficient

\section{Theoretical Analysis of Helmholtz Silencer}

\subsection{Structure and Fundamental Principles}

Figure 1 illustrates the structure of the MHS. Several chokes are inserted into a closed cylindrical vessel, dividing the vessel into several cylindrical volumes. Figure 2 illustrates the physical model of this type of silencer. The fluid in each choke acts as a mass because of the inertial effect of the fluid, and each divided cylindrical volume acts as a spring because of the capacitive effects of the fluid. The viscous friction in each choke provides a dashpot effect. For Fig.1, the resonance phenomena of a three-degree-of-freedom system, which is based on the three spring-mass system with a common base shown in Fig.2, are utilized for the attenuation of pressure pulsation. The resonant frequencies of the $3 \mathrm{HS}$ can be freely tuned by appropriately adjusting the length and diameter of each choke and associated cylindrical volume. 


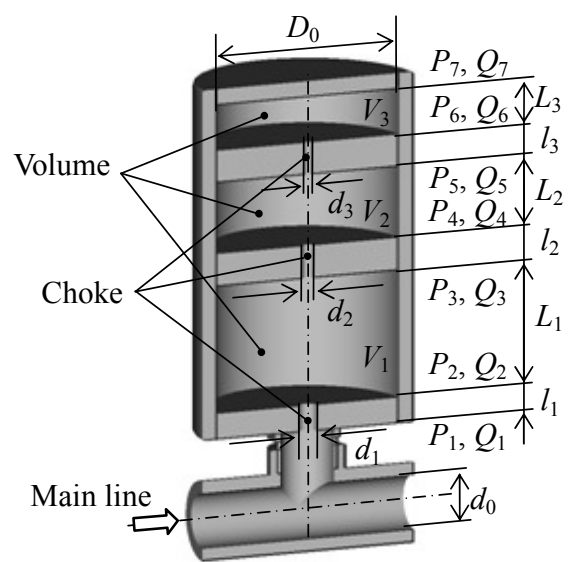

Fig. 1 Structure of the multiple-degreeof-freedom Helmholtz silencer

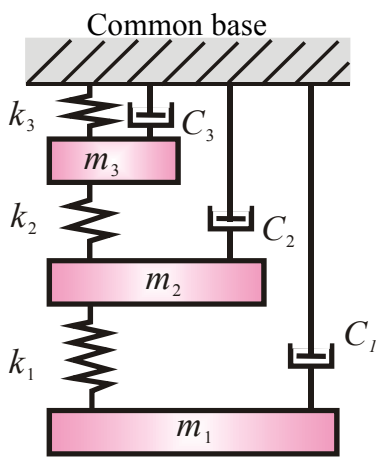

Fig. 2 Physical model of the present silencer

\subsection{Lumped Parameter Model of Three-Degree-of-Freedom Helmholtz Silencer}

Helmholtz silencer utilizes the resonant phenomena to attenuate the pressure pulsation in hydraulic circuits. Therefore, the maximum attenuation performance is achieved only at the resonant frequency and its attenuation performance will fall sharply if the frequency is apart from the resonant frequency. Helmholtz silencer is generally analyzed by the distributed parameter model and the lumped parameter model which are based on the dynamic characteristics of fluid lines ${ }^{(9)}$-(11). The distributed parameter model can model the resonance phenomena of the Helmholtz silencer more precisely than the lumped parameter model. Consequently, the distributed parameter model has to be used to design the MHS (2),

(3). However, at the initial design stage of the MHS, the distributed parameter model, which is described in Appendix A, is not able to estimate the silencer dimensions in advance because the equation is complex. Thus, the lumped parameter model has an advantage that the model equation generally includes the comprehensible relationship between the silencer dimensions and attenuation performance. For this reason, it is worth examining the lumped parameter model for the MHS in this chapter.

The attenuation performance of the silencer is evaluated using the resonant frequency $f_{n}$ and transmission loss $T L$. Transmission loss is an inherent measure of the attenuation performance of a hydraulic silencer under the anechoic termination condition; it is defined as the ratio of the incident and transmitted pulsation wave energy of the silencer and is given by the following equation ${ }^{(12)}$.

$$
T L=10 \log _{10}\left|\frac{W_{i}}{W_{t}}\right|=20 \log _{10}\left\{\frac{1}{2}\left|2+\frac{Z_{c}}{Z_{h}}\right|\right\}
$$

In this equation, $Z_{c}$ is the characteristic impedance of the main line

$$
Z_{c}=\frac{4 \rho c \xi(s)}{\pi d_{0}^{2}}
$$

where $s$ is a Laplace operator, $c$ is the speed of sound, and $d_{0}$ is the diameter of the main line. The complex coefficient for the unsteady viscous friction effect $\xi(s)$ is expressed by ${ }^{(13)}$

$$
\xi(s) \cong 1+\sqrt{\frac{4 v}{d_{0}^{2} s}}+\frac{4 v}{d_{0}^{2} s}
$$


where $v$ is the kinematic viscosity of the oil. In Eq.(1), $Z_{h}$ is the entrance impedance of the silencer, which is the ratio of the pressure pulsation to flow pulsation through the first choke $\left(P_{1} / Q_{1}\right)$. In order to obtain the attenuation performance of the silencer from Eq.(1), the entrance impedance of the silencer must be determined. For the $3 \mathrm{HS}$, the equation of motion for the $i$ th choke can be expressed as follows:

$$
\rho a_{i} l_{i} \frac{d}{d t}\left(\frac{Q_{2 i-1}(t)}{a_{i}}\right)=a_{i}\left\{P_{2 i}(t)-P_{2 i-1}(t)\right\}-a_{i} \frac{128 \mu l_{i}}{\pi d_{i}^{4}} Q_{2 i-1}(t)
$$

where subscript $i$ is 1 to $3, a_{i}$ is the cross sectional area of the $i$ th choke, $Q$ is the flow pulsation, $P$ is the pressure pulsation, $\rho$ is the density of the oil, and $\mu$ is the viscosity of the oil. By using Laplace transform, Eq.(4) becomes

$$
\left(m_{i} s+C_{i}\right) Q_{2 i-1}(s)=P_{2 i}(s)-P_{2 i-1}(s)
$$

The two coefficients $m_{\mathrm{i}}$ and $C_{i}$ are referred to as fluid inductance and fluid resistance, respectively, and are defined as follows.

$$
\left.\begin{array}{rl}
m_{i} & =\frac{\rho l_{i}}{a_{i}} \\
C_{i} & =\frac{128 \mu l_{i}}{\pi d_{i}^{4}}
\end{array}\right\}
$$

The continuity equation in the $i$ th volume considering the compressibility of the oil is given by

$$
Q_{2 i}(t)-Q_{2 i+1}(t)=\frac{V_{i}}{\kappa} \frac{d P_{2 i}(t)}{d t}
$$

where $V_{i}$ is the $i$ th volume, and $\kappa$ is the bulk modulus of the oil. At the end of the silencer flow pulsation, $Q_{7}=0$. By using Laplace transform, this equation becomes

$$
Q_{2 i}(s)-Q_{2 i+1}(s)=\frac{s}{k_{i}} P_{2 i}(s)
$$

In Eq.(8), spring coefficient for the $i$ th volume $k_{i}$ is defined as

$$
k_{i}=\frac{\kappa}{V_{i}}
$$

From the above equations, the inverse of the entrance impedance is described as follows:

$$
\frac{1}{Z_{h}}=\frac{Q_{1}}{P_{1}}=s \frac{M(s)}{H(s)}
$$

in this equation, $M(s)$ and $H(s)$ are given by

$$
\begin{aligned}
M(s)= & k_{1} k_{2}+k_{1} k_{3}+k_{2} k_{3}+\left(C_{3} k_{1}+C_{2} k_{2}+C_{3} k_{2}+C_{2} k_{3}\right) s \\
& +\left(C_{2} C_{3}+k_{2} m_{2}+k_{3} m_{2}+k_{1} m_{3}+k_{2} m_{3}\right) s^{2}+\left(C_{3} m_{2}+C_{2} m_{3}\right) s^{3}+m_{2} m_{3} s^{4}
\end{aligned}
$$

$$
\begin{aligned}
H(s) & =k_{1} k_{2} k_{3}+\left(C_{1} k_{1} k_{2}+C_{2} k_{1} k_{2}+C_{3} k_{1} k_{2}+C_{1} k_{1} k_{3}+C_{2} k_{1} k_{3}+C_{1} k_{2} k_{3}\right) s \\
& +\left(C_{1} C_{3} k_{1}+C_{2} C_{3} k_{1}+C_{1} C_{2} k_{2}+C_{1} C_{3} k_{2}+C_{1} C_{2} k_{3}+k_{1} k_{2} m_{1}+k_{1} k_{3} m_{1}+k_{2} k_{3} m_{1}\right. \\
& \left.+k_{1} k_{2} m_{2}+k_{1} k_{3} m_{2}+k_{1} k_{2} m_{3}\right) s^{2}+\left(C_{1} C_{2} C_{3}+C_{3} k_{1} m_{1}+C_{2} k_{2} m_{1}+C_{3} k_{2} m_{1}\right. \\
& \left.+C_{2} k_{3} m_{1}+C_{3} k_{1} m_{2}+C_{1} k_{2} m_{2}+C_{1} k_{3} m_{2}+C_{1} k_{1} m_{3}+C_{2} k_{1} m_{3}+C_{1} k_{2} m_{3}\right) s^{3} \\
& +\left(C_{2} C_{3} m_{1}+C_{1} C_{3} m_{2}+k_{1} m_{1} m_{3}+k_{2} m_{1} m_{2}+k_{3} m_{1} m_{2}+C_{1} C_{2} m_{3}+k_{1} m_{1} m_{3}\right. \\
& \left.+k_{2} m_{1} m_{3}+k_{1} m_{2} m_{3}\right) s^{4}+\left(C_{3} m_{1} m_{2}+C_{2} m_{1} m_{3}+C_{1} m_{2} m_{3}\right) s^{5}+m_{1} m_{2} m_{3} s^{6}
\end{aligned}
$$


The transmission loss of the 3HS can be obtained from Eq.(1) and Eqs.(10) - (12). As previously mentioned, the purpose of this research is to clarify the influence of each dimension on the attenuation characteristics. However, both derived lumped parameter model and distributed parameter model of the 3HS are highly complex to clarify the relationships between the dimensions, resonant frequencies, and transmission loss characteristics. Therefore, in the following section, the relationships between dimensions and attenuation characteristics are obtained using the simpler model, i.e., the lumped parameter model, for the conventional Helmholtz silencer instead of the $3 \mathrm{HS}$.

\subsection{Relationship between Dimensions and Attenuation Performance}

Figure 3 shows the structure of the conventional Helmholtz silencer. The inverse of the entrance impedance of this conventional Helmholtz silencer is given by Eq.(13). This equation can be derived from the lumped modeling procedure in the previous section.

$$
\frac{1}{Z_{h}}=s \frac{K \omega_{n}{ }^{2}}{s^{2}+2 \zeta \omega_{n} s+\omega_{n}{ }^{2}}
$$

This model includes the standard second-order lag element. $K$ is the gain constant, $\zeta$ is the damping coefficient, and $\omega_{n}$ is the resonant angular frequency. These parameters are described by the following expressions.

$$
\left.\begin{array}{c}
K=\frac{V_{h}}{\kappa} \\
\zeta=32 v \sqrt{\frac{\rho}{\pi \kappa}} \frac{\sqrt{V_{h} l_{h}}}{d_{h}^{3}} \\
\omega_{n}=\frac{1}{2} \sqrt{\frac{\pi \kappa}{\rho}} \frac{d_{h}}{\sqrt{V_{h} l_{h}}}
\end{array}\right\}
$$

where $d_{h}$ is the diameter of the choke, $l_{h}$ is the length of the choke, and $V_{h}\left(=\pi D_{h}^{2} L_{h} / 4\right)$ is the cylindrical volume. The resonant angular frequency $\omega_{n}$ can be rewritten as frequency:

$$
f_{n}=\frac{1}{4} \sqrt{\frac{\kappa}{\pi \rho}} \frac{d_{h}}{\sqrt{V_{h} l_{h}}}
$$

From these equations and Eq.(1), the attenuation performance, i.e., the resonant frequency $f_{n}$ and peak value of transmission loss $T L\left(\right.$ at $\left.f=f_{n}\right)$, can be related to the design parameters as follows:

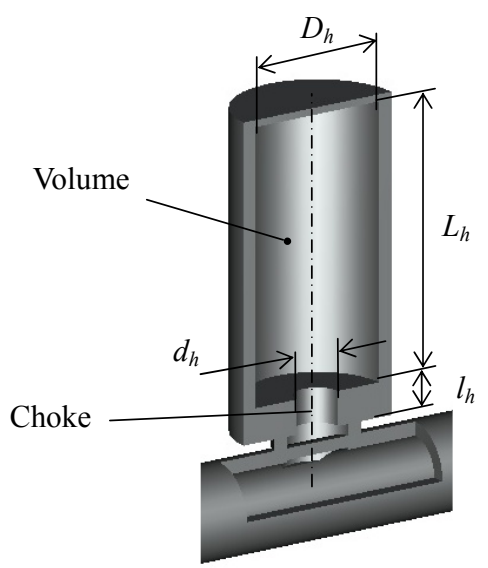

Fig. 3 Structure of the conventional Helmholtz silencer 


$$
\left.\begin{array}{l}
f_{n} \propto \frac{d_{h}}{\sqrt{V_{h} l_{h}}} \\
T L \propto \frac{d_{h}^{4}}{l_{h}}
\end{array}\right\}
$$

These relationships will be very important in the initial design of the MHS. The diameter of the choke $d_{h}$ is an important parameter for the attenuation performance of the silencer because its order is higher than those of other parameters. Interestingly, Eq.(16) indicates that the cylindrical volume $V_{h}$ is independent of the transmission loss characteristics; only the dimensions of the choke, $d_{h}$ and $l_{h}$ determine the maximum attenuation performance.

Table 1 Reference dimensions of conventional Helmholtz silencer

\begin{tabular}{|l|l|}
\hline Diameter of choke & $d_{h}=10 \mathrm{~mm}$ \\
\hline Length of choke & $l_{h}=30 \mathrm{~mm}$ \\
\hline Volume & $V_{h}=3.93 \times 10^{-4} \mathrm{~m}^{2}$ \\
\hline Diameter of cylindrical volume & $D_{h}=50 \mathrm{~mm}$ \\
\hline Length of cylindrical volume & $L_{h}=200 \mathrm{~mm}$ \\
\hline
\end{tabular}

Here the influence of changes in dimensions is investigated in detail to help ascertain ideal choke size and cylindrical volume for the Helmholtz silencer. The examples of dimensions used in the theoretical examinations are listed in Table 1. First, the cylindrical volume $V_{h}$ is varied by changing the length of cylindrical volume $L_{h}$, which is proportional to the volume change $\left(V_{h}=\pi D_{h}{ }^{2} / 4 \times L_{h}\right)$. The results are also calculated using the distributed parameter model expressed in Eq.(B.1) for comparison (see Appendix B). Because the resonant frequency $f_{n}$ and the peak value of transmission loss cannot be calculated directly from Eq.(B.1), the frequency characteristics of transmission loss is firstly calculated as shown in Fig.8. The resonant frequency $f_{n}$ and transmission loss $T L$ at $f_{n}$ is searched numerically for all changing parameters from these results. The calculated results for both models are shown in Fig.4. As expressed in Eq.(16), the cylindrical volume has no effect on the transmission loss characteristics in the lumped parameter model. It could also be said that the results of the distributed parameter model have little influence on transmission loss. The quantitative difference in transmission loss between two models is clear in Fig.4(b). This deviation will be discussed in the next section, along with the experimental results for transmission loss. Regarding to the resonant frequency $f_{n}$, the results of both models exhibit the following relationship: $f_{n} \propto 1 / \sqrt{V_{h}}$ as shown in Fig.4(a). These results imply that varying the cylindrical volume makes it possible to vary the resonant frequency without changing the peak value of transmission loss. Therefore, any mechanism that varies the volume is sufficient to realize a Helmholtz silencer with variable resonance. It should be noted that tuning the resonant frequency $f_{n}$ by varying the length of cylindrical volume $L_{h}$ has limitations, because the frequency $f_{n}$ will saturate a certain value within a practical range of volume length; the resonant frequency cannot be tuned to lower than $400 \mathrm{~Hz}$ by varying the length of cylindrical volume in case of Fig.4. Therefore, other parameter would be used to set the lower limit of the resonant frequency.

Second, the length of choke $l_{h}$ is chosen as the parameter of study. Fig.5 illustrates the influence of changes in choke length. The most notable feature of this figure is that the relationships between the length of choke $l_{h}$ and both resonant frequency $f_{n}$ and transmission 
loss $T L$ are exactly expressed as those described by Eq.(16): $f_{n} \propto 1 / \sqrt{l_{h}}$ and $T L \propto 1 / l_{h}$, respectively. Unlike the length of cylindrical volume $L_{h}$, this parameter is not appropriate for adjusting the resonant frequency $f_{n}$, since the change of transmission loss is accompanied. In order to maintain the attenuation performance for all the object frequency range, the peak value of transmission loss is desired to be at a constant.

Finally, the influence of choke diameter $d_{h}$ is examined. Fig. 6 displays the results for a quarter wavelength side branch silencer, in addition to those for the two models. The side branch resonator is a branch pipe installed in the main flow line ${ }^{(14)}$. The pressure pulsation is attenuated by utilizing the reflections of pulsation waves in the branch pipe. The fluid wave in the side branch is reflected only at the closed end of the branch, and its reflection ratio is independent of frequency. Consequently the resonant phenomenon occurs at

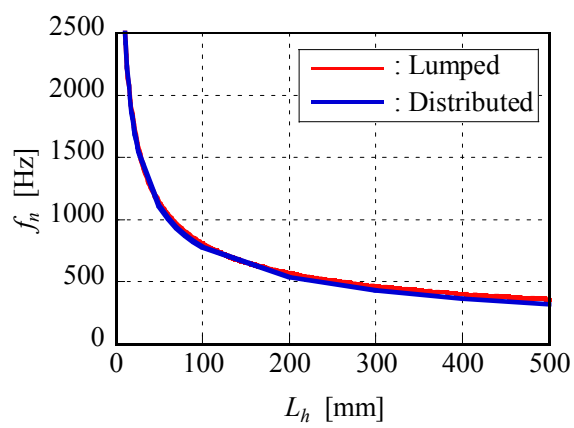

(a) Resonant frequency $f_{n}$

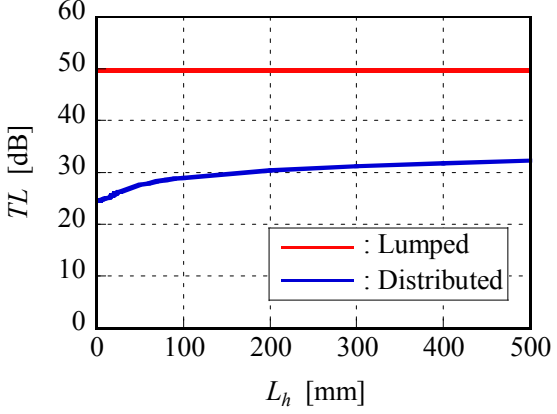

(b) Transmission loss $T L$ at $f_{n}$

Fig. 4 Influence of the length of cylindrical volume $L_{h}$

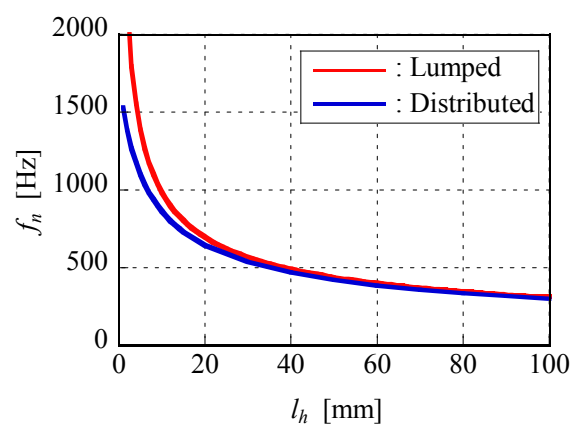

(a) Resonant frequency $f_{n}$

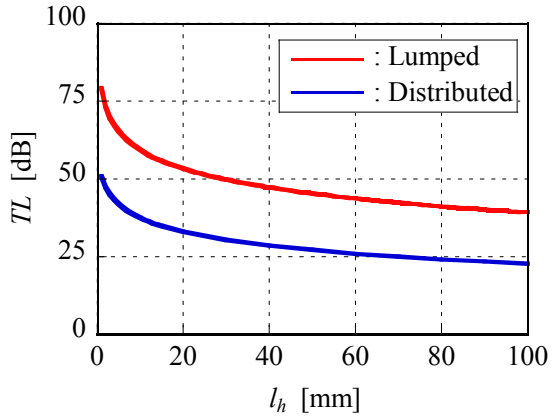

(b) Transmission loss $T L$ at $f_{n}$

Fig. 5 Influence of the length of choke $l_{h}$

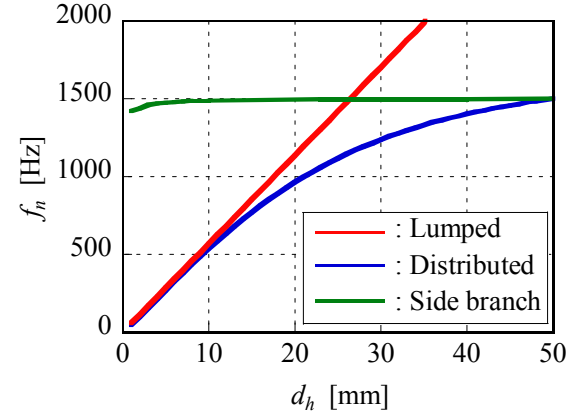

(a) Resonant frequency $f_{n}$

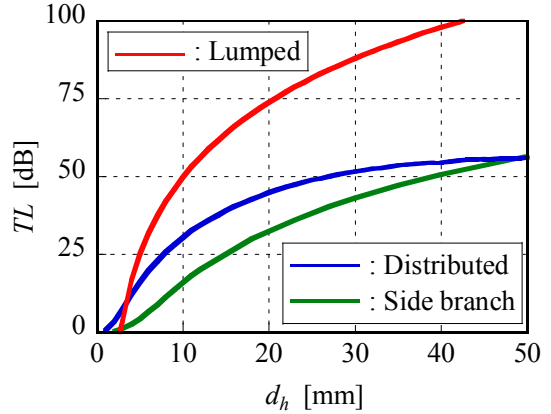

(b) Transmission loss $T L$ at $f_{n}$

Fig. 6 Influence of the diameter of choke $d_{h}$ 
frequency corresponding to the quarter wavelength of the branch length. This means the resonant frequency of the side branch silencer is dependent only on the length of branch pipe. In case of Fig.6, the resonant frequency becomes $f_{n} \cong 1560 \mathrm{~Hz}$ since the length of side branch is $l_{h}+L_{h}=220 \mathrm{~mm}\left(f_{n}=c /\left\{4\left(l_{h}+L_{h}\right)\right\}\right)$. The diameter of the side branch is set to the diameter of the choke $d_{h}$. It can be seen from Fig. 6 that this parameter is suitable for setting the lower limit of the resonant frequency $f_{n}$ because it can be tuned from $0 \mathrm{~Hz}$. The resonant frequency of the distributed parameter model (blue line) begins to deviate from the lumped parameter model at $d_{h}=10 \mathrm{~mm}$, and this deviation increases with diameter $d_{h}$. It transitions from the lumped parameter model to the side-branch model. Concerning about the transmission loss $T L$ at $f_{n}$, the quantitative difference between the lumped parameter model and distributed parameter model also becomes significant by increasing the diameter of choke $d_{h}$. These deviations can be explained from the fact that the Helmholtz silencer begins to act as a side-branch resonator when the diameter of the choke is close to the diameter of the cylindrical volume. Subsequently, when the diameters of the choke and cylindrical volume are the same, the attenuation characteristics reflect only those of the side-branch resonator. In practice, however, the choke diameter is rarely larger than the diameter of the cylindrical volume. The maximum diameter of the choke will equal the diameter of the main line.

\section{Experimental Examinations}

\subsection{Test Helmholtz Silencer}

The aim of this research is to obtain the design criteria for the 3HS that would allow it to be applied to hydraulic systems operating at variable speeds. The theoretical analysis in Section 2 clarified that the choke diameter $d_{h}$ is an important parameter in the design of a Helmholtz silencer with the desired attenuation performance. In this section, an experimental investigation of the attenuation characteristics of the conventional Helmholtz silencer is conducted in order to verify the theoretical relationship between the resonant frequency $f_{n}$ and choke diameter $d_{h}$.

The dimensions of the provided Helmholtz silencer are the same as those applied in the theoretical examination in the previous section. The volume is set to $V_{h}=3.93 \times 10^{-4} \mathrm{~m}^{2}$ by setting the length as $L_{h}=200 \mathrm{~mm}$ and diameter as $D_{h}=50 \mathrm{~mm}$ of the cylindrical volume. The test Helmholtz silencer is manufactured such that the choke part is replaceable. The choke diameters are set to $d_{h}=4,7,10,13,16,20,23$, and $27 \mathrm{~mm}$. The maximum choke diameter of $d_{h}=27 \mathrm{~mm}$ is equal to the diameter of the main line. The length of choke is $l_{h}=30 \mathrm{~mm}$. The effect of fluid inertia through the choke must be taken into account as an open-end correction. Therefore, the length of the replaceable choke $l_{\mathrm{c}}$ is determined in accordance with the following equation, which is a function of the choke diameter $d_{h}$ :

$$
l_{c}=l_{h}-\beta d_{h}
$$

where the open-end correction coefficient $\beta$ is determined as $\beta=8 /(3 \pi)^{(15)}$.

\subsection{Experimental Apparatus}

The transmission loss of hydraulic silencers described in Eq.(1) can also be calculated by the transfer matrix coefficients of the silencer. The transfer matrix has $2 \times 2$ elements that express the pressure and flow wave propagation characteristics of hydraulic components. In this paper, the experimentally obtained transfer matrix is used to identify the transmission loss of the test Helmholtz silencer. The method adopted for the measurement of the transfer matrix is described in the reference ${ }^{(12)}$; therefore, only an outline of the experimental apparatus is presented in this study. The hydraulic test circuit and instruments necessary for 
the experimental method are depicted in Fig.7. The input pressure pulsation is generated by a radial piston pump with 5 pistons, which produces wide band flow pulsation up to $f=2000$ Hz. The hydraulic circuit is arranged in accordance with the experimental method ${ }^{(12)}$. Four piezoelectric pressure transducers are equipped upstream and downstream of the test Helmholtz silencer. These transducers are mounted on the block such that their diaphragms are flush with the inner wall of the pipe. The lengths between each pressure transducer are listed in Table 2. The average discharge pressure is set to $10 \mathrm{MPa}$ by operating the loading valves. The working oil used in this test is ISO VG-32 mineral oil and the temperature is maintained at $40^{\circ} \mathrm{C}$ during the measurements. Other important specifications are listed in Table 2.

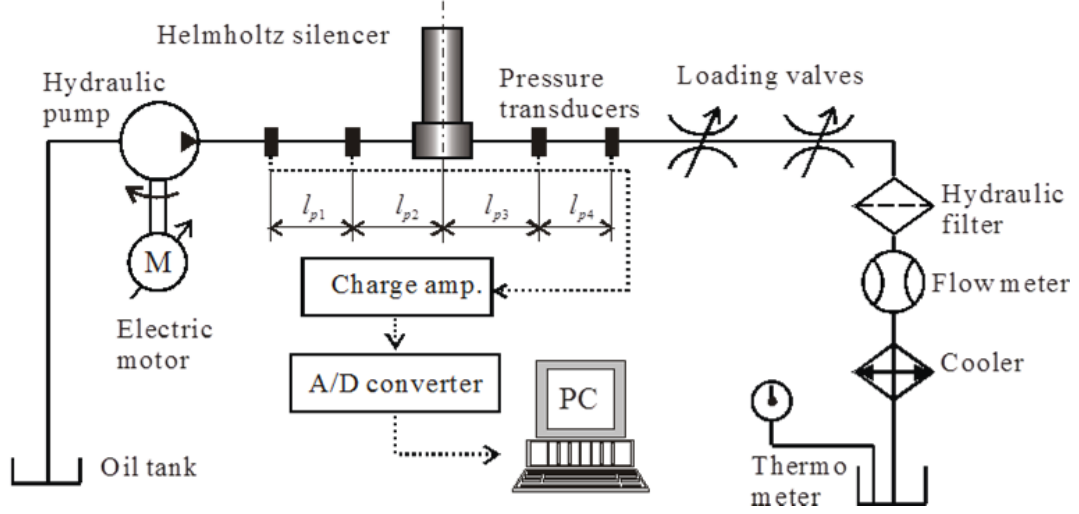

(a) Hydraulic circuit and measurement instruments

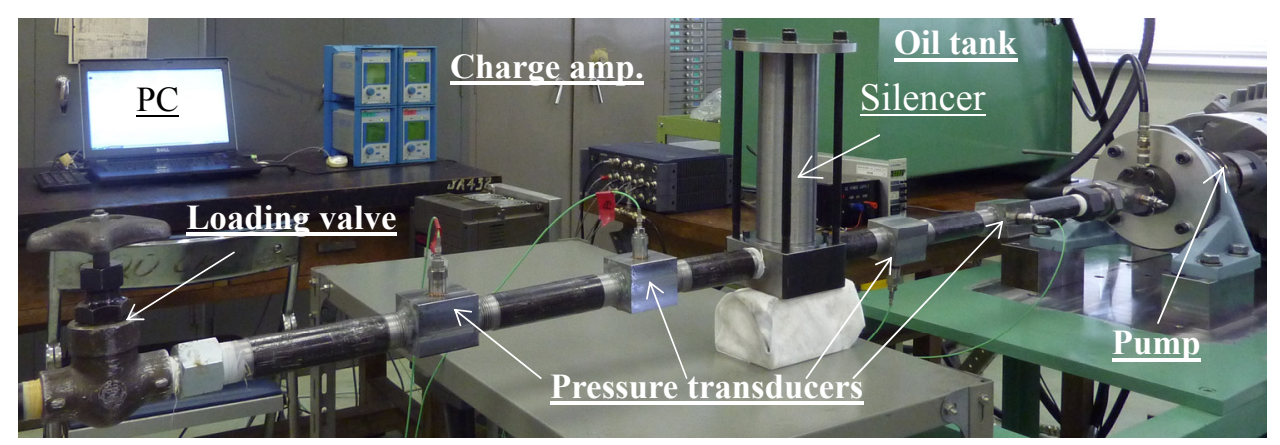

(b) Image of experimental apparatus

Fig. 7 Experimental apparatus

Table 2 Specifications of the experimental apparatus

\begin{tabular}{|l|l|}
\hline Speed of sound & $c=1380 \mathrm{~m} / \mathrm{s}$ \\
\hline Diameter of main line & $d_{0}=27.2 \mathrm{~mm}$ \\
\hline \multirow{4}{*}{$\begin{array}{l}\text { Length between each pressure } \\
\text { transducers and the silencer }\end{array}$} & $l_{p 1}=230 \mathrm{~mm}$ \\
\cline { 2 - 2 } & $l_{p 2}=200 \mathrm{~mm}$ \\
\cline { 2 - 2 } & $l_{p 3}=200 \mathrm{~mm}$ \\
\cline { 2 - 2 } & $l_{p 4}=230 \mathrm{~mm}$ \\
\hline Mean discharge pressure & $P_{d}=10 \mathrm{MPa}$ \\
\hline Bulk modulus of oil & $\kappa=1.66 \mathrm{GPa}$ \\
\hline Kinematic viscosity of oil & $v=3.2 \times 10^{-5} \mathrm{~m}^{2} / \mathrm{s}$ \\
\hline Density of oil & $\rho=870 \mathrm{~kg} / \mathrm{m}^{3}$ \\
\hline
\end{tabular}




\subsection{Experimental Results and Considerations}

The experimental results for transmission loss at $d_{h}=4,10,16$, and $27 \mathrm{~mm}$ are shown in Fig.8(a) - (d) along with the calculated results for the two models; namely, the lumped parameter model and distributed parameter model. It is obvious from these figures that changing the choke diameter significantly influences the attenuation characteristics such as the resonant frequency $f_{n}$ and peak value of transmission loss. In Fig.8(a), the calculated resonant frequency $f_{n}$ is similar for both models. The theoretical examinations in the previous section, shown in Fig.6(a), suggested that the two models show similar attenuation characteristics when the choke diameter $d_{h}$ is small. Then, the disparity between the two models increases with $d_{h}$. Figure $8(\mathrm{a})-(\mathrm{d})$ confirms that the distributed parameter model agrees well with the measured transmission loss. This is primarily because the inertial effect

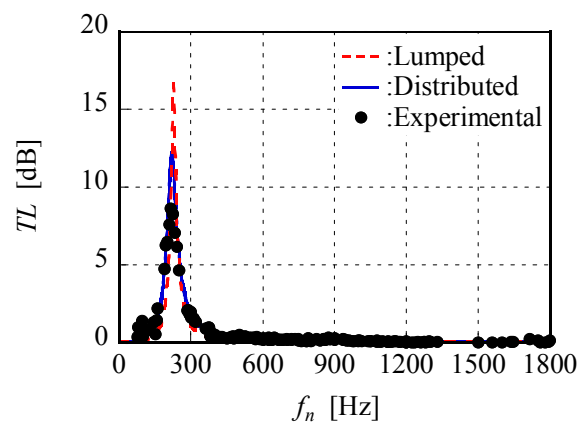

(a) $d_{h}=4 \mathrm{~mm}$

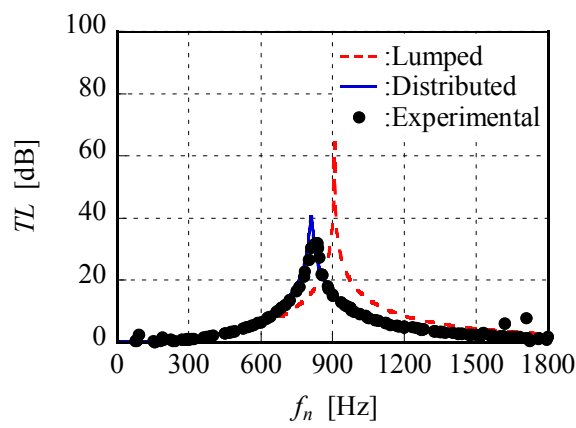

(c) $d_{h}=16 \mathrm{~mm}$

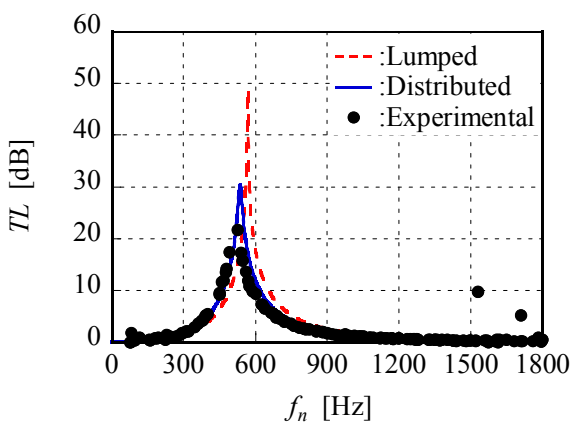

(b) $d_{h}=10 \mathrm{~mm}$

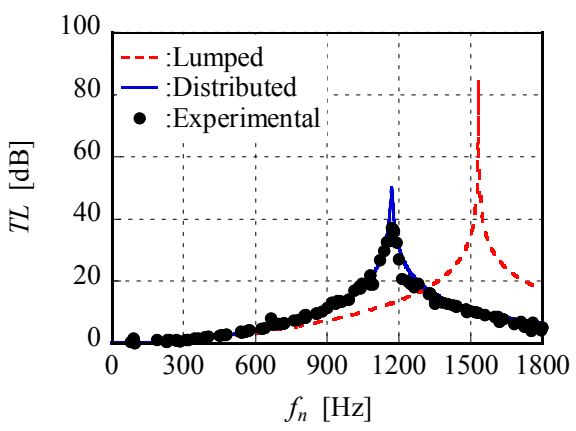

(d) $d_{h}=27 \mathrm{~mm}$

Fig. 8 Experimental results of transmission loss

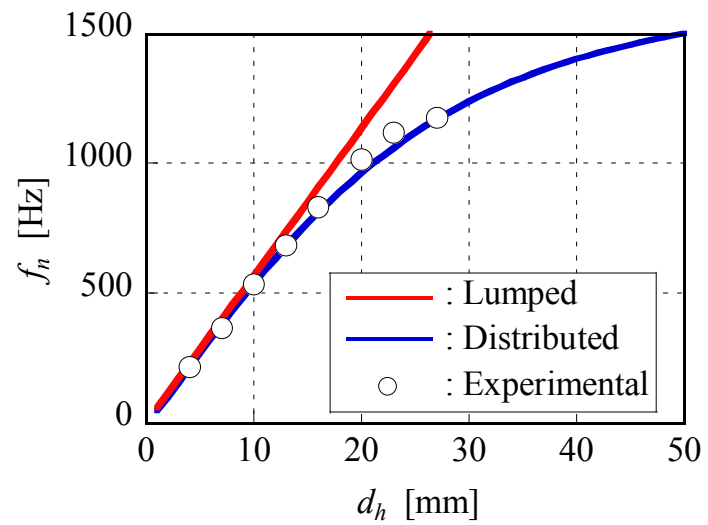

Fig. 9 Influence of the choke diameter $d_{h}$ on the resonant frequency $f_{n}$ 
of the cylindrical volume cannot be neglected and the lumped parameter model takes into account only the compressibility effect of the cylindrical volume. There is a difference between the peak transmission loss at the resonant frequency for the distributed parameter model and experimental results. However, this deviation appears to have little relation to the choke diameter $d_{h}$ and is instead due to the inaccuracy of the transmission loss model, particularly at the resonant frequency. The average ratio of the experimental result to the result from the distributed parameter model for their peak values is around 0.7.

The experimental results for the resonant frequency $f_{n}$ are obtained from the measured transmission loss. Figure 9 shows the influence of choke diameter $d_{h}$ on the resonant frequency $f_{n}$; the experimental results agree well with the distributed parameter model. If the choke diameter $d_{h}$ can be increased above $27 \mathrm{~mm}$ in the experimental study, the maximum resonant frequency $f_{n}$ can be predicted to be close to the attenuation frequency of the side-branch silencer, as discussed in the previous section. This increase in the choke diameter $d_{h}$ will be accompanied by an enlargement of the diameter of the main line.

The important findings from the experimental results for the design of a Helmholtz silencer are as follows. Because the experimental results show better agreement with the distributed parameter model than the lumped parameter model, the distributed parameter model should be used to design the Helmholtz silencer. Nevertheless, the lumped parameter model can be also utilized to approximately design the silencer because it is expressed in simple form in Eq.(16), which provides an idea of how the design parameters relate to the attenuation performance. We must take into account this particular tendency such that the resonant frequency $f_{n}$ converges to the attenuation frequency of the side-branch silencer as the diameter increases.

\section{Design of Multiple-Degree-of-Freedom Helmholtz Silencer}

In this section, a prototype design of a $3 \mathrm{HS}$ with variable resonance ability is presented. Its main feature is that the dimensions of the $3 \mathrm{HS}$ must be continuously changed by adding some variable resonance mechanism. The qualitative relationship expressed in Eq.(16) is utilized for the initial design of this variable-type $3 \mathrm{HS}$, even though there will be a discrepancy between the resonant frequency of the $3 \mathrm{HS}$ and resonant frequency estimated from Eq.(16).

The design features of the Helmholtz silencer that have been clarified are as follows. Changing the choke diameter $d_{h}$ only makes it possible to vary the resonant frequency $f_{n}$ from $0 \mathrm{~Hz}$, whereas the other parameters have less of an effect on the lowest frequencies attainable. Varying the volume size, i.e., the length of volume $L_{h}$, allows variation in the resonant frequency $f_{n}$ without changing the peak value of transmission loss. Accordingly, the length of volume is an appropriate parameter for adapting the variable mechanism in order to vary the resonant frequency $f_{n}$. It is also preferable from a mechanical point of view because the mechanism for adjusting the choke diameter and length is too complex in a high-pressure hydraulic system. Therefore, the $3 \mathrm{HS}$ with variable resonance can be realized by employing a variable mechanism such that two choke parts with constant dimension move inside the cylindrical volume in a manner similar to the mechanism of a hydraulic cylinder.

The qualitative relationship expressed by Eq.(16) gives an idea of how choke diameters should be determined. The target attenuation frequencies are usually the fundamental, second, and third harmonic components of the pumping frequency. The $3 \mathrm{HS}$ has three resonant frequencies that must coincide with these harmonics. The first, second and third chokes have a significant influence on the design of third, second and fundamental frequency respectively. Therefore, the diameter of the third choke should be firstly determined by considering both fundamental frequency and desired attenuation value of 
transmission loss. In this paper, the diameters of two chokes are designed to be double and threefold of the smallest diameter. It should be noted that the length of chokes can be shorter as reducing the diameter if the desired value of transmission loss is constant. The shorter choke design will contribute to the compactness of the $3 \mathrm{HS}$ with variable resonant ability.

The following two characteristics represent the desired attenuation performance of the prototype design in this report.

(a) The fundamental pumping frequency varies from $125 \mathrm{~Hz}$ to $400 \mathrm{~Hz}$. Accordingly, the desired resonant frequencies are from $f_{r .1}{ }^{*}=125 \mathrm{~Hz}, f_{r .2}{ }^{*}=250 \mathrm{~Hz}, f_{r .3}{ }^{*}=375 \mathrm{~Hz}$ to $f_{r .1}{ }^{*}=400$ $\mathrm{Hz}, f_{r .2}{ }^{*}=800 \mathrm{~Hz}, f_{r .3}{ }^{*}=1200 \mathrm{~Hz}$, respectively.

(b) The peak values of transmission loss at three resonant frequencies are $T L=15 \mathrm{~dB}$ for all pump rotational speed ranges, i.e., $125 \mathrm{~Hz} \leq f_{r .1}{ }^{*} \leq 400 \mathrm{~Hz}$.

From the above consideration based on the qualitative relationship expressed by Eq.(16), the initial design was determined as [1] the configuration of the silencer is three constant chokes and three volumes with the variable lengths, [2] the diameters of two chokes are double and threefold of the smallest diameter.

First, three constant chokes are obtained by the numerical optimum design method for the desired resonant frequency $f_{r .1}{ }^{*}=250 \mathrm{~Hz}\left(T L\left(f_{r .1}\right)=15 \mathrm{~dB}\right)$. The reason why this frequency is chosen for the three constant chokes is because it is close to the central value of the objective frequencies $125 \mathrm{~Hz}$ to $400 \mathrm{~Hz}$. When it comes to manufacture these chokes, the choke holes are generally processed by drilling and then the size of choke diameters should match the size of a drill. This means the size of choke diameters are excluded from the design variables in the numerical optimum design method. The diameter of the first choke is also constrained by the diameter of the main hydraulic line because the diameter of choke has to be smaller than the diameter of the main line due to its installation. In this research the diameters of the three chokes are set to $d_{1}=6 \mathrm{~mm}, d_{2}=4 \mathrm{~mm}$, and $d_{3}=2 \mathrm{~mm}$, taking into account the above considerations. The diameter of the cylindrical volume is $D_{0}=50 \mathrm{~mm}$. The design variables, objective function, and constraint condition are expressed as:

$$
\begin{array}{ll}
\text { Design variables: } & \mathbf{X}={ }^{t}\left\{l_{1}, l_{2}, l_{3}, L_{1}, L_{2}, L_{3}\right\} \\
\text { Objected function: } & f(\mathbf{X})=\left|f_{r .1}-f_{r .1}{ }^{*}\right|+\left|f_{r .2}-f_{r .2}{ }^{*}\right|+\left|f_{r .3}-f_{r .3}{ }^{*}\right| \\
\text { Constraint condition: } & T L\left(f_{r .1}\right)=T L\left(f_{r .2}\right)=T L\left(f_{r .3}\right)=15 \mathrm{~dB}
\end{array}
$$

The purpose of this calculation is to search the unknown lengths so that the resonant frequency $f_{r . i}$ of the silencer coincides with the desired value $f_{r . i}{ }^{*}$. For the optimum design algorithm, Powell's conjugate directions method is adopted to search for these design variables ${ }^{(16)}$. The lengths are then searched as $l_{1}=16.1 \mathrm{~mm}, l_{2}=17.3 \mathrm{~mm}$, and $l_{3}=11.1 \mathrm{~mm}$. These diameter and length values become the dimensions of the three constant chokes.

Finally, the unknown lengths of the three cylindrical volumes $\left(L_{1}, L_{2}\right.$, and $\left.L_{3}\right)$ for the varying frequency range are obtained also using the numerical optimum design method. In this calculation, the design variable decreases as $L_{1}, L_{2}$, and $L_{3}$. The ratios of transmission loss at the resonant frequency $\varepsilon$ are constrained while searching the unknown lengths (as $\varepsilon=1.0$ ). The following equations represent the design variables, objective function, and constraint condition.

$$
\text { Design variables: } \quad \mathbf{X}={ }^{t}\left\{L_{1}, L_{2}, L_{3}\right\}
$$


Constraint condition: $\frac{T L\left(f_{r .2}\right)}{T L\left(f_{r .1}\right)}=\frac{T L\left(f_{r .3}\right)}{T L\left(f_{r .1}\right)}=\varepsilon$

Figure 10 illustrates the searched lengths of cylindrical volumes $L_{1}, L_{2}$, and $L_{3}$ for varying resonant frequencies. The numerical optimum design calculation was done at every $50 \mathrm{~Hz}$ within the target desired resonant frequency range. The horizontal axis in Fig. 10 represents the desired fundamental resonant frequency $f_{r .1}{ }^{*}$. The second and third resonant frequencies are double and threefold of $f_{r .1}{ }^{*}$. It is clear that the lengths of the three cylindrical volumes continuously vary with changes in the resonant frequency. Therefore, it is confirmed that varying the lengths of the three cylindrical volumes with three constant chokes makes it possible to achieve variable resonance at the desired frequencies. The transmission loss characteristic for the present silencer is shown in Fig.11. The figure clearly indicates the design variables are well searched for the given constraint condition $\varepsilon=1.0$ within all the frequency range $125 \mathrm{~Hz}<f_{r .1}{ }^{*}<400 \mathrm{~Hz}$. The peak values of transmission loss at each resonance frequencies are almost constant when the lengths of cylindrical volumes are varied. As discussed before for the conventional Helmholtz silencer, the change of these lengths has also little influence on the transmission loss for the $3 \mathrm{HS}$.

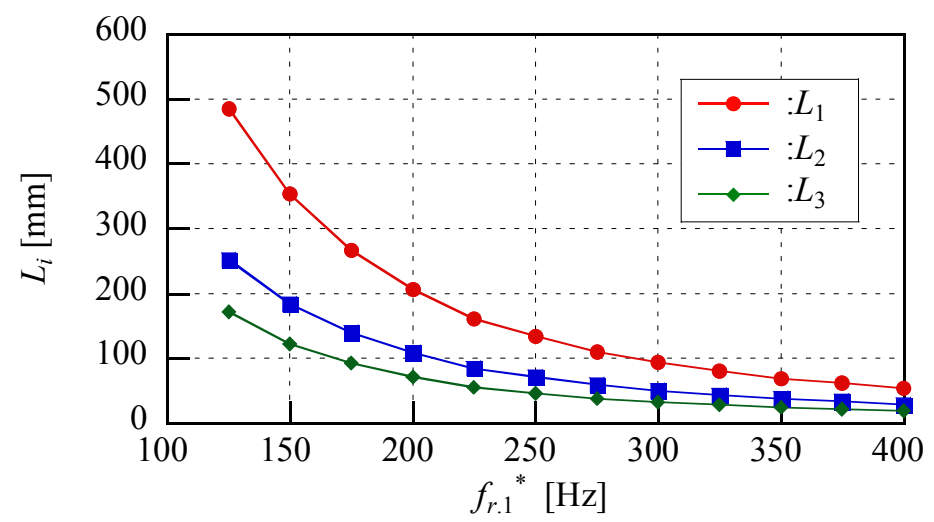

Fig. 10 Designed length of volume for the present silencer

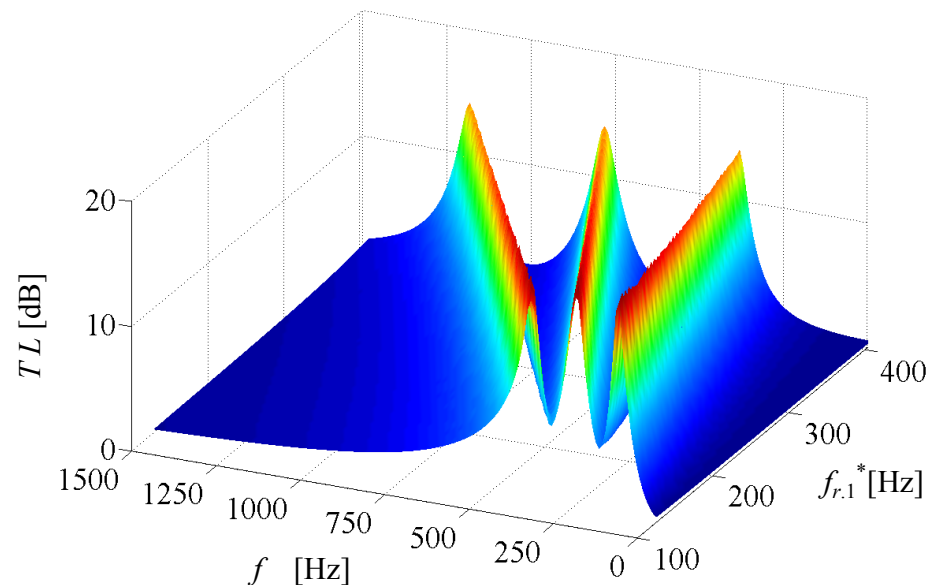

Fig. 11 Transmission loss characteristics of the present silencer 


\section{Conclusions}

The final aim of this project is the development of a multiple-degree-of-freedom Helmholtz silencer that can be applied to hydraulic systems operated under variable pump speed conditions. In this study, in order to establish the design criteria of the MHS, the attenuation characteristics were theoretically and experimentally investigated with respect to the design parameters for the conventional Helmholtz silencer. The results of this study clarified the relationship between the design dimension parameters (such as the length and diameter) and transmission loss characteristics. This relationship and the knowledge obtained from the parametric study were utilized for the initial design of the 3HS with variable resonant ability. The $3 \mathrm{HS}$, which satisfies the desired attenuation performance, was well designed by employing the three constant chokes and variable volumes. Therefore, it was confirmed that these information can be useful for the initial design of the MHS. In the next report, a test $3 \mathrm{HS}$ equipped with a variable resonant mechanism will be experimentally examined to ascertain its variable resonant attenuation characteristics and evaluate its practical performance.

\section{Appendix}

\section{A: Distributed Parameter Model of Three-Degree-of-Freedom Helmholtz Silencer}

Consider the Helmholtz silencer with three chokes shown in Fig.1. Assuming the fluid flow in the cylindrical volume to be two-dimensional, compressible and laminar flow, the Laplace transfer matrix between the upstream and the downstream for the $i$ th divided cylindrical volume $\mathbf{A}_{i}$ can be obtained from the Navier-Stokes equation and the continuity equation considering compressibility as follows ${ }^{(13)}$.

$$
\mathbf{A}_{i}=\left[\begin{array}{cc}
\cosh \left\{\frac{\xi_{V}(s) s}{c} L_{i}\right\} & Z_{c . V} \sinh \left\{\frac{\xi_{V}(s) s}{c} L_{i}\right\} \\
\frac{1}{Z_{c . V}} \sinh \left\{\frac{\xi_{V}(s) s}{c} L_{i}\right\} & \cosh \left\{\frac{\xi_{V}(s) s}{c} L_{i}\right\}
\end{array}\right]
$$

where $L_{i}$ is the length of the $i$ th divided cylindrical volume. $\xi_{V}(s)$ and $\mathrm{Z}_{c . V}$ are the complex coefficient for unsteady viscous friction effect and the characteristic impedance of the cylindrical volume respectively. These parameters are expressed as follows.

$$
\begin{aligned}
& \xi_{V}(s) \cong 1+\sqrt{\frac{4 v}{D_{0}^{2} s}}+\frac{4 v}{D_{0}^{2} s} \\
& Z_{c, V}=\frac{4 \rho c \xi_{V}(s)}{\pi D_{0}^{2}}
\end{aligned}
$$

The transfer matrix of the $i$ th chokes are also given by the same equation as Eq.(A.1). But this equation can be simplified like Eq.(A.4) since the length of choke $l_{i}$ is generally far shorter than the wave length.

$$
\mathbf{B}_{i}=\left[\begin{array}{cc}
1 & \frac{4 \rho l_{i} s \xi_{N . i}(s)^{2}}{\pi d_{i}^{2}} \\
0 & 1
\end{array}\right]
$$

where $\xi_{N . i}(s)$ is the complex coefficient for unsteady viscous friction effect of the $i$ th divided choke. This is the same equation as Eq.(A.2) with using the $i$ th divided choke diameters $d_{i}$ instead of $D_{0}$.

For the Helmholtz silencer with three chokes, the transfer matrix between the inlet and 
the closed-end of cylindrical vessel can be described by the following equation with the transfer matrix $\mathbf{C}$.

$$
\mathbf{C}=\mathbf{B}_{1} \cdot \mathbf{A}_{1} \cdot \mathbf{B}_{2} \cdot \mathbf{A}_{2} \cdot \mathbf{B}_{3} \cdot \mathbf{A}_{3} \equiv\left[\begin{array}{ll}
C_{1.1} & C_{1.2} \\
C_{2.1} & C_{2.2}
\end{array}\right]
$$

Once the transfer matrix $\mathbf{C}$ is obtained, the inverse of the entrance impedance can be also given by the following equation.

$$
\frac{1}{Z_{r}}=\frac{C_{2.1}}{C_{1.1}}
$$

\section{B: Distributed Parameter Model of Conventional Helmholtz Silencer}

For the conventional Helmholtz silencer shown in Fig.3, the transfer matrix between the inlet and the closed-end can be obtained by the dot product of the transfer matrices for a single choke (Eq.(A.4)) and single cylindrical volume (Eq.(A.1)). The inverse of the entrance impedance is derived from the relationship of Eq.(A.6) as follows.

$$
\frac{1}{Z_{r}}=\frac{1}{Z_{c . h} \operatorname{coth}\left\{\frac{\xi_{V . h}(s) s}{c} L_{h}\right\}+\frac{4 \rho l_{h} s \xi_{N . h}(s)^{2}}{\pi d_{h}^{2}}}
$$

where $Z_{c . h}$ is the characteristic impedance of the cylindrical volume for the conventional Helmholtz silencer. $\xi_{V . h}(s), \xi_{N . h}(s)$ are the complex coefficients for unsteady viscous friction effect of the cylindrical volume and choke. These parameters are expressed by the same equations as Eqs.(A.2) and (A.3).

\section{References}

(1) Henderson, R., Quieter Fluid Power Handbook, (1981), BHRA Fluid Engineering.

(2) Ichiyanagi, T. and Kojima, E., Development and Optimum Design Method of Multi Degree of Freedom Type Helmholtz Resonator for Hydraulic Pulsation Attenuation, Transactions of the Japan Fluid Power System Society, Vol.32, No.1 (2001), pp.13-20.

(3) Washio, S., Takahashi, S., Mukaibatake, K. and Yamaguchi, S., Oil Hydraulic Pulsation Filters of Helmholtz Resonator Type, Journal of the Japan Hydraulics and Pneumatics Society, Vol.24, No.6 (1993), pp.729-735.

(4) Chanaud, R. C., Effects of Geometry on the Resonance Frequency of Helmholtz Resonators, Journal of Sound and Vibration, Vol.178, No.3 (1994), pp.337-348.

(5) Ronald, L. P., Effect of Orifice Geometry on Helmholtz Resonator Excitation by Grazing Flow, The American Institute of Aeronautics and Astronautics Journal, Vol.28, No.1 (1989), pp.60-65.

(6) Selamet, A., Dicley, N. S. and Novak, J. M., Theoretical, Computational and Experimental investigation of Helmholtz Resonators with Fixed Volume: Lumped versus Distributed analysis, Journal of Sound and Vibration, Vol.187, No.2 (1995), pp.358-367.

(7) Selamet, A. and Lee, I., Helmholtz Resonator with Extended Neck, Journal of the Acoustic Society of America, Vol.113, No.4-1 (2003), pp.1975-1985.

(8) Yoshioka, M., Application of a Series-Line Acoustic Resonator to Measurements, Transactions of the Japan Society of Mechanical Engineers, Series C, Vol. 63, No. 615 (1997), pp.3911-3918.

(9) Ishihara, T., Ichikawa, T., Kaneko, T. and Takenaka, T., Hydraulic Engineering Handbook, (1972), pp.154-156, Asakura Publishing Co., Ltd. (in Japanese) 
(10) The Japan Hydraulics and Pneumatics Society, Hydraulics and Pneumatics Handbook New Edition, (1989), p.72, Ohmsha, Ltd. (in Japanese)

(11) Blackburn, J. F., Fluid Power Control, (1960), pp.134-137, The M. I. T Press.

(12) Kojima, E. and Ichiyanagi, T., Generation and Propagation of Fluid-borne Pressure Ripple in Fluid Power Systems Caused a Pump (2nd Report, Experimental Determination of Transfer Matrix Parameters of Hydraulic Silences and Assessment of its Attenuation Performance), Transactions of the Japan Society of Mechanical Engineers, Series B, Vol. 61, No. 583 (1995), pp. 1014-1022.

(13) Brown, F. T. and Nelson, S. E., Step Response of Liquid Lines with Frequency Dependent Effects of Viscosity, Journal of Basic Engineering, Transactions of ASME, Series D, Vol. 87, No.2 (1965), pp. 504-510.

(14) The Japan Society of Mechanical Engineers, Flow Induced Vibrations; Classification and Lessons from Practical Experiences, (2003), pp.190-191, Gihodo Shuppan Co.,Ltd.

(15) Washio, S. and Konishi, T., Research on Wave Phenomena in Hydraulic Lines: 8th Report, Theoretical Investigation on End Correction Problems, (Part 1, Flow Analysis in Terms of Reactive Properties), Transactions of the Japan Society of Mechanical Engineers, Series B, Vol.47, No.421 (1981), pp. 1757-1764.

(16) Powell, M. J. D., An Efficient Method for Finding the Minimum of a Function of Several Variables without Calculating Derivatives, The Computer Journal, Vol.7 (1964), pp.155-162. 